\title{
EULAR recommendations for the management of systemic lupus erythematosus with neuropsychiatric manifestations: report of a task force of the EULAR standing committee for clinical affairs
}

\author{
G K Bertsias, ${ }^{1} \mathrm{~J}$ P A loannidis, ${ }^{2} \mathrm{M}$ Aringer, ${ }^{3} \mathrm{E}$ Bollen, ${ }^{4}$ S Bombardieri, ${ }^{5}$ I N Bruce, ${ }^{6}$ \\ R Cervera, ${ }^{7}$ M Dalakas, ${ }^{8}$ A Doria, ${ }^{9} \mathrm{~J} \mathrm{G} \mathrm{Hanly,}{ }^{10}$ T W J Huizinga, ${ }^{11} \mathrm{D}$ Isenberg, ${ }^{12}$ \\ C Kallenberg, ${ }^{13} \mathrm{~J}$ C Piette, ${ }^{14} \mathrm{M}$ Schneider, ${ }^{15} \mathrm{~N}$ Scolding, ${ }^{16} \mathrm{~J}$ Smolen, ${ }^{17} \mathrm{~A}$ Stara, ${ }^{18}$ \\ I Tassiulas, ${ }^{19} \mathrm{M}$ Tektonidou, ${ }^{20} \mathrm{~A}$ Tincani, ${ }^{21} \mathrm{M}$ A van Buchem, ${ }^{22} \mathrm{R}$ van Vollenhoven, ${ }^{23}$ \\ M Ward, ${ }^{24}$ C Gordon, ${ }^{25}$ D T Boumpas ${ }^{1}$
}

\begin{abstract}
Additional data (supplementary tables) are published online only. To view these files please visit the journal online (http://ard.bmj. com)

For numbered affiliations see end of article
\end{abstract}

\section{Correspondence to}

Dr D T Boumpas, Departments of Internal Medicine and Rheumatology, University of Crete School of Medicine, 71 003, 1 Voutes Street, Heraklion, Greece: boumpasd@med.uoc.gr

Accepted 25 June 2010

Published Online First 19 August 2010

\begin{abstract}
Objectives To develop recommendations for the diagnosis, prevention and treatment of neuropsychiatric systemic lupus erythematosus (NPSLE) manifestations. Methods The authors compiled questions on prevalence and risk factors, diagnosis and monitoring, therapy and prognosis of NPSLE. A systematic literature search was performed and evidence was categorised based on sample size and study design.
\end{abstract}

Results Systemic lupus erythematosus (SLE) patients are at increased risk of several neuropsychiatric manifestations. Common (cumulative incidence $>5 \%$ ) manifestations include cerebrovascular disease (CVD) and seizures; relatively uncommon (1-5\%) are severe cognitive dysfunction, major depression, acute confusional state (ACS), peripheral nervous disorders psychosis. Strong risk factors lat least fivefold increased risk) are previous or concurrent severe NPSLE (for cognitive dysfunction, seizures) and antiphospholipid antibodies (for CVD, seizures, chorea). The diagnostic work-up of suspected NPSLE is comparable to that in patients without SLE who present with the same manifestations, and aims to exclude causes unrelated to SLE. Investigations include cerebrospinal fluid analysis (to exclude central nervous system infection), EEG (to diagnose seizure disorder), neuropsychological tests (to assess cognitive dysfunction), nerve conduction studies (for peripheral neuropathy) and MRI (T1/T2, fluid-attenuating inversion recovery, diffusion-weighted imaging, enhanced T1 sequence). Glucocorticoids and immunosuppressive therapy are indicated when NPSLE is thought to reflect an inflammatory process (optic neuritis, transverse myelitis, peripheral neuropathy, refractory seizures, psychosis, ACS) and in the presence of generalised lupus activity. Antiplatelet/anticoagulation therapy is indicated when manifestations are related to antiphospholipid antibodies, particularly thrombotic CVD. Conclusions Neuropsychiatric manifestations in SLE patients should be first evaluated and treated as in patients without SLE, and secondarily attributed to SLE and treated accordingly.

In 1999, the American College of Rheumatology (ACR) research committee published a set of case definitions for neuropsychiatric systemic lupus erythematosus (NPSLE) manifestations, ${ }^{1}$ which involve the central and the peripheral nervous system and that range from overt manifestations such as stroke, seizures and psychosis, to more subtle abnormalities of cognitive function (see supplementary table S1, available online only). Multiple pathological mechanisms are implicated in NPSLE, including antiphospholipid or other autoantibodymediated vascular or neuronal injury, intrathecal production of inflammatory mediators and accelerated atherosclerosis. Despite substantial advances in the understanding of lupus, NPSLE continues to pose diagnostic and therapeutic challenges to practising physicians. The indicated diagnostic work-up remains unclear, therapies are empiric, and the prognosis after an neuropsychiatric event is often difficult to determine. We sought to develop recommendations for the management of systemic lupus erythematosus (SLE) patients presenting with neuropsychiatric manifestations using an evidencebased approach followed by expert consensus.

\section{METHODS}

The European League Against Rheumatism (EULAR) standardised operating procedures were followed and the expert committee created a list of research questions that were further edited for literature search (table 1 and supplementary file, available online only). A systematic search of PubMed was performed using an array of relevant terms, ${ }^{2}$ and all English language publications up to January 2009 were considered. Evidence was graded based on the design and validity of available studies and the strength of the statements was graded A-D (table 2). Following discussions, the committee arrived at 15 final statements (table 3). Each member of the committee rated their agreement with each statement, based on the research evidence presented and their own expertise. The guidelines fulfil all 23 items of the Appraisal of Guidelines Research and Evaluation (AGREE) instrument.

\section{RESULTS}

\section{General NPSLE}

Prevalence of NPSLE

Most (50-60\%) NPSLE events occur at disease onset or within the first year after SLE onset, 
commonly $(40-50 \%)$ in the presence of generalised disease activity. Data from cohort studies indicate a cumulative incidence of NPSLE of 30-40\% (supplementary table S2, available online only). Manifestations such as headache, mood disorders, anxiety and mild cognitive dysfunction are common, but do not usually reflect overt central nervous system (CNS) lupus activity. By excluding these manifestations and polyneuropathy without electrophysiological confirmation, reported NPSLE frequency decreases by half and the specificity of the ACR nomenclature increases from $46 \%$ to $93 \%$. ${ }^{3}$

\section{Risk factors for NPSLE}

Risk factors consistently associated with NPSLE events include (supplementary table S3, available online only): (1) general SLE activity or damage, especially for seizure disorders and severe cognitive dysfunction ${ }^{5-7}$; (2) previous events or other concurrent NPSLE manifestations ${ }^{8-10}$; and (3) antiphospholipid antibodies (persistently positive moderate-to-high anticardiolipin or anti $\beta 2$-glycoprotein IgG/IgM titres or the lupus anticoagulant), especially for cerebrovascular disease (CVD), ${ }^{6}{ }^{9}$ seizure disorder, ${ }^{5} 8$ moderate-to-severe cognitive dysfunction, ${ }^{711}$ myelopathy ${ }^{12}$ and movement disorder. ${ }^{11}$

\section{Diagnosis of NPSLE}

The evaluation of SLE patients with (new) signs or symptoms suggestive of neuropsychiatric disease is comparable to that in

\section{Table 1 Selected questions on NPSLE for the literature search}

Prevalence and risk factors
What is the prevalence of neuropsychiatric manifestations in SLE patients and
how much more common are they compared to people without SLE?
Are any of the classic risk factors for the neuropsychiatric manifestations more
common in SLE patients?
Are there any risk factors that are specific to SLE patients only?
Screening, diagnosis and monitoring
Should the screening and diagnostic work-up and monitoring for SLE patients
with neuropsychiatric manifestations differ from that in non-SLE patients and if
so, what particular tests should be applied and in which settings or indications?
Prevention and treatment
Are there any treatment interventions that need to be specifically considered
in SLE patients with neuropsychiatric manifestations, and if so, with what
diagnostic documentation and with what threshold of initiation, dosage, duration,
contraindications?
Prognosis
Is prognosis different in SLE patients with neuropsychiatric manifestations
compared to non-SLE patients regarding the manifestation itself and the disease in
general?

non-SLE patients who present with the same manifestations, ${ }^{2}$ and initially aims to exclude secondary causes such as infections, metabolic or endocrine disturbances and adverse drug reactions (supplementary table S4, available online only).

Cerebrospinal fluid (CSF) examination (including PCR for herpes simplex virus (HSV) and JC virus as indicated) may help to exclude CNS infection in patients with fever or other signs and symptoms suggestive of infection; mild CSF abnormalities are common (40-50\%) but are not specific to the NPSLE manifestations. EEG studies may help to diagnose underlying seizure disorder. Neuroimaging may detect NPSLE involvement and exclude other (neurosurgical, infectious) causes. The imaging technique of choice is MRI (T1/T2-weighted imaging, a fluidattenuating inversion recovery sequence, diffusion-weighted imaging (DWI) and a gadolinium-enhanced T1-weighted sequence). The average sensitivity of MRI in active NPSLE is $57 \%$ (64\% in major vs $30 \%$ in minor NPSLE, $76 \%$ in focal vs $51 \%$ in diffuse NPSLE). The most frequent pathological pattern is small punctate hyperintense T2-weighted focal lesions in subcortical and periventricular white matter (WM), usually in the frontal-parietal regions. Unfortunately, these MRI lesions are also present in many patients without neuropsychiatric manifestations (specificity 60-82\%). ${ }^{13-15}$

When conventional MRI is normal or does not provide an explanation for the signs and symptoms, advanced neuroimaging may be performed. Modalities to be considered (based on availability and local expertise) include quantitative MRI (magnetic resonance spectroscopy, ${ }^{16} 17$ magnetisation transfer imaging, ${ }^{18} 19$ diffusion tensor $\mathrm{MRI},{ }^{20}$ perfusion-weighted imaging) or radionuclide brain scanning (single photon emission computed tomography (SPECT), ${ }^{21} 22$ or positron emission tomography ${ }^{23}$ ). These imaging studies may reveal additional WM and grey matter abnormalities, which, however, have modest specificity for NPSLE.

\section{Management of NPSLE}

General management involves the correction of aggravating factors and symptomatic therapy when appropriate (supplementary table S5, available online only). Specific therapy depends upon the nature of the underlying process (inflammatory or thrombotic). The committee concluded that in selected cases differentiation between these processes may not be feasible and in some patients both mechanisms may be operant. When NPSLE is thought to reflect an inflammatory/neurotoxic process (especially aseptic meningitis, optic neuritis, transverse myelitis, peripheral neuropathy, refractory seizures, psychosis, acute confusional state; ACS) and in the presence of generalised

Table 2 Category of evidence and strength of statements rating scales

\section{Category of evidence}

\section{Diagnostic/prognostic studies}

1 The available evidence is strong and includes consistent results from well-designed, well-conducted studies

2 The available evidence is sufficient to determine effects, but confidence in the estimate is constrained by such factors as: the number, size, or quality of individual studies, inconsistency of findings across individual studies, limited generalisability of findings

3 The available evidence is insufficient due to the limited number or size of studies, important flaws in study design or methods, inconsistency of findings across individual studies, gaps in the chain of evidence, lack of information on important outcomes

\section{Intervention studies \\ At least one RCT or meta-analysis of RCT Controlled (non-randomised) studies}

Descriptive studies, such as comparative studies, correlation studies, or case-control studies

\section{Strength of statements}

A

B

C

D
Based on category 1 evidence

Based on category 2 evidence, or extrapolated recommendations from category 1 evidence Based on category 3 evidence, or extrapolated recommendations from category 2 evidence Expert opinion or standard of care

$\mathrm{RCT}$, randomised controlled trial. 
Table 3 EULAR recommendations for the management of NPSLE

\begin{tabular}{ll} 
Statement & $\begin{array}{l}\text { Category of } \begin{array}{l}\text { Strength of } \\
\text { statement }\end{array} \text { Agreement } \\
\text { score }\end{array}$ \\
\hline
\end{tabular}

\section{General NPSLE}

NPSLE

Neuropsychiatric events may precede, coincide, or follow the diagnosis of SLE but commonly (50-60\%) occur within the first year

Cumulative incidence

Common (5-15\% cumulative incidence) manifestations include CVD and seizures; Relatively uncommon (1-5\%): severe cognitive dysfunction, major depression, ACS and peripheral nervous disorders; Rare $(<1 \%)$ are psychosis, myelitis, chorea, cranial neuropathies and aseptic meningitis.

Risk factors

Strong (fivefold increase) risk factors consistently associated with primary NPSLE are generalised SLE activity, previous severe NPSLE manifestations (especially for cognitive dysfunction and seizures), and antiphospholipid antibodies (especially for CVD, seizures, chorea)

Diagnostic work-up

In SLE patients with new or unexplained symptoms or signs suggestive of neuropsychiatric disease, initial diagnostic work-up should be similar to that in non-SLE patients presenting with the same manifestations

Depending upon the type of neuropsychiatric manifestation, this may include lumbar puncture and CSF analysis (primarily to exclude CNS infection), EEG, neuropsychological assessment of cognitive function, NCS, and neuroimaging (MRI) to assess brain structure and function

The recommended MRI protocol (brain and spinal cord) includes conventional MRI sequences (T1/T2, FLAIR), DWI, and gadolinium-enhanced $\mathrm{T} 1$ sequences

Therapy

Glucocorticoids and immunosuppressive therapy are indicated for neuropsychiatric manifestations felt to reflect an immune/ inflammatory process (eg, ACS, aseptic meningitis, myelitis, cranial and peripheral neuropathies and psychosis) following exclusions of non-SLE-related causes

Antiplatelet/anticoagulation therapy is indicated when manifestations are related to antiphospholipid antibodies, particularly in thrombotic CVD

The use of symptomatic therapies (eg, anticonvulsants, antidepressants) and the treatment of aggravating factors (eg, infection, hypertension and metabolic abnormalities) should also be considered

Antiplatelet agents may be considered for primary prevention in SLE patients with persistently positive, moderate or high, antiphospholipid antibody titres

Specific NPSLE disorders

CVD

Atherosclerotic/thrombotic/embolic CVD is common, haemorrhagic stroke is rare, and stroke caused by vasculitis is very rare in SLE patients; accordingly, immunosuppressive therapy is rarely indicated

Long-term anticoagulation should be considered in patients with stroke who fulfil the classification criteria for antiphospholipid syndrome for secondary prevention of recurrent stroke which commonly occurs

Cognitive dysfunction

Mild or moderate cognitive dysfunction is common in SLE but severe cognitive impairment resulting in functional compromise is relatively uncommon and should be confirmed by neuropsychological tests in collaboration with a clinical neuropsychologist when available

Management of both SLE and non-SLE-associated factors as well as psycho-educational support may prevent further

deterioration of cognitive dysfunction; progressive cognitive decline develops only in a minority of patients

Seizure disorder

Single seizures are common in SLE patients and have been related to disease activity. Chance of recurrence is comparable to that in the general population

The diagnostic work-up aims to exclude structural brain disease and inflammatory or metabolic conditions and includes MR and EEG

In the absence of MRI lesions related to seizures and definite epileptic abnormalities on EEG following recovery from the seizure, withholding of AED after a single seizure should be considered. Long-term anti-epileptic therapy may be considered for recurrent seizures

For most patients without generalised disease activity, immunosuppressive therapy is not indicated for prevention of recurrences or control of refractory seizures

Anticoagulation may be considered in patients with antiphospholipid antibodies

Movement disorders (chorea)

In addition to symptomatic therapy for persistent symptoms (dopamine antagonists), antiplatelet agents may be considered in SLE patients with antiphospholipid antibodies

Glucocorticoids/immunosuppressive and/or anticoagulation therapy may be considered in severe cases when generalised disease activity and/or thrombotic manifestations are present

2
2
1

Major depression and psychosis

Major depression attributed to SLE alone is relatively uncommon while psychosis is rare; although steroid-induced psychosis may occur this is very rare

There is no strong evidence to support the diagnostic utility of serological markers or brain imaging in major depression Glucocorticoids and immunosuppressive therapy may be considered in SLE-associated psychosis, especially in presence of generalised disease activity 
Table 3 Continued

\begin{tabular}{|c|c|c|c|}
\hline Statement & $\begin{array}{l}\text { Category of } \\
\text { evidence }\end{array}$ & $\begin{array}{l}\text { Strength of } \\
\text { statement }\end{array}$ & $\begin{array}{l}\text { Agreement } \\
\text { score }\end{array}$ \\
\hline \multicolumn{4}{|l|}{ Myelopathy } \\
\hline The diagnostic work-up includes gadolinium-enhanced MRI and cerebrospinal fluid analysis & 2 & D & 9.5 \\
\hline Maintenance therapy with less intensive immunosuppression to prevent recurrence may be considered & 3 & D & 9.3 \\
\hline \multicolumn{4}{|l|}{ Optic neuritis is commonly bilateral in SLE } \\
\hline $\begin{array}{l}\text { Optic neuritis needs to be distinguished from ischaemic optic neuropathy, which is usually unilateral, especially in patients with } \\
\text { antiphospholipid antibodies }\end{array}$ & 3 & D & 9.3 \\
\hline $\begin{array}{l}\text { Glucocorticoids (intravenous methylprednisolone) alone or in combination with immunosuppressive agents should be } \\
\text { considered, but failures are common }\end{array}$ & 1 & A & 9.1 \\
\hline \multicolumn{4}{|l|}{ Peripheral neuropathy } \\
\hline $\begin{array}{l}\text { Peripheral neuropathy often co-exists with other neuropsychiatric manifestations and is diagnosed with electromyography and } \\
\text { NCS }\end{array}$ & 3 & D & 9.1 \\
\hline
\end{tabular}

ACS, acute confusional state; AED, anti-epilectic drug; CNS, central nervous system; CSF, cerebrospinal fluid; CVD, cerebrovascular disease; DWI, diffusion-weighted imaging; FLAIR, fluid-attenuating inversion recovery sequence; NCS, nerve conduction studies; NPSLE, neuropsychiatric systemic lupus erythematosus; SLE, systemic lupus erythematosus.

lupus activity, management includes glucocorticoids alone or in combination with immunosuppressants (azathioprine or cyclophosphamide). ${ }^{24} 25$ In severe NPSLE refractory to standard immunosuppressive therapy, among other treatments, plasma exchange, ${ }^{26} 27$ intravenous immunoglobulin, ${ }^{28-30}$ and rituximab (anti-CD20 monoclonal antibody) ${ }^{31}$ have been used.

Antiplatelet and/or anticoagulation therapy is recommended for NPSLE related to antiphospholipid antibodies, especially for thrombotic CVD. Anticoagulation may be superior to antiplatelet therapy for secondary prevention of arterial events (including stroke/transient ischaemic attack; TIA) in antiphospholipid antibody syndrome (APS). ${ }^{32-35}$ Antiplatelet/anticoagulation therapy has also been used in antiphospholipid-associated ischaemic optic neuropathy and chorea, as well as in myelopathy refractory to immunosuppressive therapy. ${ }^{36-38}$ Data from cohort studies, ${ }^{39-41}$ but not from a randomised controlled trial (RCT), ${ }^{42}$ support a potential benefit from antiplatelet agents in the primary prevention of CVD (and other thrombotic events) in SLE patients with persistently positive, moderate-to-high titres of antiphospholipid (HR 0.98-0.99 per month of aspirin therapy).

\section{Specific NPSLE manifestations}

Headache

Although headache is frequently reported by SLE patients, several studies and a meta-analysis of epidemiological data ${ }^{43}$ found no evidence of an increased prevalence or a unique type of headache in SLE. Caution is needed to exclude aseptic or septic meningitis, sinus thrombosis (especially in patients with antiphospholipid antibodies), cerebral or subarachnoid haemorrhage. In the absence of high-risk features from the medical history and the physical examination (including fever or concomitant infection, immunosuppression, presence of antiphospholipid, use of anticoagulants, focal neurological signs, altered mental status, meningismus and generalised SLE activity), headache alone in a SLE patient requires no further investigation beyond the evaluation, if any, that would have been performed for non-SLE patients.

\section{Cerebrovascular disease}

Ischaemic stroke and/or TIA comprise over $80 \%$ of CVD cases, whereas CNS vasculitis is rare. CVD occurs commonly $(50-60 \%)$ in the context of high disease activity and/or damage; other strong risk factors are persistently positive moderate-to- high titres of antiphospholipid antibodies, heart valve disease, systemic hypertension and old age.

In acute stroke, MRI/DWI excludes haemorrhage, assesses the degree of brain injury, and identifies the vascular lesion responsible for the ischaemic deficit. Magnetic resonance angiography, CT angiography, or conventional angiography may help to characterise the vascular lesions and detect brain vasculature aneurysms in subarachnoid haemorrhage.

The acute management of SLE stroke or TIA is similar to that in the general population. A stroke specialist consultation is necessary to identify patients who are candidates for thombolytic or surgical therapy; unless contraindicated, aspirin should be initiated. Secondary prevention includes tight control of cardiovascular risk factors, antiplatelet therapy and carotid endarterectomy when indicated. Generalised lupus activity may be controlled with glucocorticoids and/or immunosuppressive therapy. In patients with persistently positive moderate-to-high titres of antiphospholipid antibodies, chronic oral anticoagulation therapy should be considered. ${ }^{32} 33$ Two RCT of 114 and 109 patients with mixed (primary and SLE-related) APS have demonstrated no superiority of high-intensity warfarin (target international normalised ratio (INR) 3.1-4.0) over moderateintensity warfarin (target INR 2.0-3.0) for secondary thromboprophylaxis, but the risk of minor bleeding was increased in the high-intensity arm (28\% vs $11 \%) .{ }^{44} 45$ Conversely, retrospective studies that included larger numbers of patients with arterial thrombosis or stroke concluded that high-intensity anticoagulation may be more effective without increasing the risk of major bleeding. ${ }^{32} 3346$ Accordingly, and based on the results of a systematic review of epidemiological studies, ${ }^{35}$ some experts recommend that SLE patients with ischaemic stroke fulfilling the criteria for APS should receive long-term anticoagulation treatment with a target INR of 3.0-4.0,47 48 but this is still a matter of debate. ${ }^{49}$

\section{Cognitive dysfunction}

Most SLE patients have a mild-to-moderate degree of cognitive dysfunction with an overall benign course, and severe cognitive dysfunction develops only in 3-5\%.7 50 Most commonly affected domains are attention, visual memory, verbal memory, executive function and psychomotor speed.

ACR has proposed a $1 \mathrm{~h}$ battery of neuropsychological tests for diagnosing cognitive dysfunction in SLE (sensitivity $80 \%$, 
specificity $81 \%) .{ }^{1}$ The computer-based automated neuropsychological assessment metrics system has also been used. Indications for brain MRI include: age less than 60 years, rapid unexplained or moderate-to-severe cognitive decline, recent and significant head trauma, new onset of other neurological symptoms or signs, and development of cognitive dysfunction in the setting of immunosuppressive or antiplatelet/anticoagulation therapy. Cerebral atrophy, the number and size of WM lesions, and cerebral infarcts have been correlated with the severity of cognitive dysfunction. ${ }^{7}$ 51-53

Management involves treatment of any exacerbating causes, especially anxiety and depression, and control of cardiovascular risk factors. Although a single study has reported a favourable association between regular aspirin use and cognitive function in older diabetes patients with SLE, ${ }^{54}$ the efficacy of antiplatelet therapy has not been established. Psycho-educational group interventions have demonstrated improvements in memory function and the ability to perform daily activities (mean cognitive symptoms inventory score improved from 2.1 to 1.7 ). ${ }^{55} 56$ Glucocorticoids and/or immunosuppressive therapy may be considered to control concurrent SLE or other NPSLE activity. ${ }^{57} 58$ APS patients with cognitive dysfunction may benefit from anticoagulation therapy but evidence comes from a single, uncontrolled study. ${ }^{59}$

\section{Seizure disorders}

Most seizures in SLE represent single isolated events; recurrent seizures (epilepsy) are less common (12-22\%) but have a significant impact on morbidity and mortality. Patients can experience generalised tonic-clonic seizures (67-88\%) or partial (complex) seizures

EEG abnormalities are common (60-70\%) in SLE patients with seizure disorder, but typical epileptiform EEG patterns are only present in $24-50 \%$ and are predictive of seizure recurrence (positive predictive value $73 \%$, negative predictive value $79 \%) .{ }^{860} \mathrm{MRI}$ can identify structural lesions causally related to seizure disorder and may reveal abnormalities such as cerebral atrophy $(40 \%)$ and WM lesions (50-55\%). CSF examination is only useful to exclude infection.

Anti-epileptic drug (AED) therapy is not necessary in patients with single or infrequent seizures, unless high-risk features for recurrences are present, such as two or more unprovoked seizures occurring with at least $24 \mathrm{~h}$ interval, serious brain injury, brain MRI structural abnormalities causally linked to seizures, focal neurological signs, partial seizure and epileptiform EEG. Approximately a quarter of SLE patients will require a second AED to control seizure activity. ${ }^{60}$ If seizures are thought to reflect an acute inflammatory event or if a concomitant lupus flare is present, glucocorticoids alone or in combination with immunosuppressive therapy may be given. The combination of pulse intravenous methylprednisolone and intravenous cyclophosphamide has shown effectiveness in refractory seizures in the context of generalised lupus activity. ${ }^{25}$

\section{Movement disorders}

Chorea (irregular, involuntary and jerky movements involving any part of the body in random sequence) is the best documented movement disorder in SLE, and has been associated with antiphospholipid antibodies and/or APS. Brain imaging should be considered when other focal neurological signs are present or to exclude secondary causes of chorea. Most patients (55-65\%) experience a single episode of chorea that subsides within days to a few months. Symptomatic therapy with dopamine antagonists is usually effective and glucocorticoids in combination with immunosuppressive agents (azathioprine, cyclophosphamide) may be used to control NPSLE disease activity. Antiplatelet and/ or anticoagulation therapy is administered in antiphospholipidpositive patients, especially when other antiphospholipid/APSrelated manifestations are present. ${ }^{36} 6162$

\section{Acute confusional state}

ACS is characterised by acute onset, fluctuating level of consciousness with decreased attention. Patients should be extensively evaluated for underlying precipitating conditions, especially infections and metabolic disturbances. CSF examination is recommended to exclude CNS infection and EEG may help diagnose underlying seizure disorder. Brain imaging is indicated if the patient has focal neurological signs, history of head trauma or malignancy, fever, or when the initial diagnostic work-up has failed to reveal any obvious cause of the ACS. Brain SPECT is sensitive (93\%) and may help monitor response to treatment. 31

Management requires addressing and correcting the underlying causes. Drug treatment with haloperidol or atypical antipsychotics is used only when other interventions are ineffective in controlling agitation and an underlying cause of ACS has been excluded. A combination of glucocorticoids with immunosuppressive agents is effective in most patients (response rates up to $70 \%$ ). ${ }^{25} 6364$ Plasma exchange therapy (synchronised with intravenous cyclophosphamide $)^{26} 27$ and rituximab have been used in refractory cases.

\section{Psychiatric disorders}

Lupus psychosis is characterised by delusions (false beliefs refuted by objective evidence) or hallucinations (perceptions in the absence of external stimuli). Corticosteroid-induced psychiatric disease occurs in $10 \%$ of patients treated with prednisone $1 \mathrm{mg} / \mathrm{kg}$ or more and it manifests primarily as mood disorder (93\%) rather than psychosis. ${ }^{65}$ Although anti-ribosomal-P antibodies have been associated with psychiatric SLE in prospective studies, ${ }^{66} 67$ a meta-analysis has reported limited diagnostic accuracy (sensitivity $25-27 \%$, specificity $75-80 \%$ ). ${ }^{68}$

Brain MRI has modest sensitivity (50-70\%) and specificity (40-67\%) for lupus psychosis, and should be considered when additional neurological symptoms or signs are present. Brain SPECT identifies perfusion deficits in severe cases (80-100\%) and residual hypoperfusion during clinical remission correlates with future relapse. ${ }^{69}$

Management involves antidepressive and/or antipsychotic agents as indicated. Biofeedback-assisted cognitive behavioural treatment has a favourable impact on depressive symptoms. ${ }^{70}$ In generalised SLE activity, the combination of glucocorticoids and immunosuppressive therapy (usually cyclophosphamide, followed by maintenance with azathioprine) results in a significant improvement (60-80\% response) although relapses may occur (up to $50 \%$ ). ${ }^{64} 71-73$ In refractory cases, rituximab has caused a rapid significant improvement of psychiatric manifestations. ${ }^{31}$ Most psychiatric episodes resolve within 2-4 weeks and only $20 \%$ of SLE patients develop a chronic mild psychotic disorder.

\section{Myelopathy}

SLE myelopathy presents as rapidly evolving transverse myelitis but ischaemic/thrombotic myelopathy can also occur. Patients may present with signs of grey matter (lower motor neuron) dysfunction (flaccidity and hyporeflexia) or WM (upper motor neuron) dysfunction (spasticity and hyperreflexia); the latter can be associated more with neuromyelitis optica (NMO) and antiphospholipid. ${ }^{74}$ Other major NPSLE manifestations are present in one third of cases, with optic neuritis being the most common (21-48\%). Contrast-enhanced spinal cord MRI is 
useful to exclude cord compression and to detect T2-weighted hyperintense lesions (70-93\%). The involvement of more than three spinal cord segments indicates longitudinal myelopathy. This finding may be further investigated with determination of serum NMO IgG (aquaporin) antibodies, which help diagnose co-existing NMO. ${ }^{75}$ Brain MRI should be performed when other NPSLE symptoms or signs co-exist and in the differential diagnosis of demyelinating disorders (supplementary table S6, available online only). Mild-to-moderate CSF abnormalities are common (50-70\%) but non-specific and microbiological studies are important to exclude infectious myelitis.

Although an intensely inflammatory CSF resembling meningitis (bacterial or HSV) necessitates antimicrobial/antiviral therapy, high-dose glucocorticoids may be given early while awaiting MRI confirmation, and continued if infection has been ruled out. The combination of intravenous methylprednisolone and intravenous cyclophosphamide can be effective in SLE myelitis if used promptly, within the first few hours, and neurological response paralleled by MRI improvement occurs within a few days to 3 weeks. Relapses are common (50-60\%) during corticosteroid dose reduction, underscoring the need for maintenance immunosuppressive therapy. Plasma exchange therapy has been used in severe cases 262776 and anticoagulation therapy in antiphospholipid-positive myelopathy with good results. ${ }^{37}$ 38 Factors associated with severe neurological deficit include extensive spinal cord MRI lesions, reduced muscle strength or sphincter dysfunction at presentation, antiphospholipid antibodies, and delay ( $>2$ weeks) in the initiation of therapy. ${ }^{76} 77$

\section{Cranial neuropathy}

Most frequent cranial neuropathies involve the eighth, the oculomotor (third, fourth and sixth), and less commonly the fifth and seventh nerves. Other neurological conditions, such as brainstem stroke and meningitis, should be excluded. Optic neuropathy includes inflammatory optic neuritis and ischaemic/thrombotic optic neuropathy. Funduscopy may reveal optic disc oedema (30-40\%) and visual field examination may show central or arcuate defects. Visual-evoked potentials may detect bilateral optic nerve damage before it is clinically apparent. Fluoroangiography should be performed when vaso-occlusive retinopathy is suspected. Co-existing transverse myelitis or seizure disorder may suggest an underlying inflammatory basis, while optic neuropathy with an altitudinal field defect, associated with antiphospholipid antibodies, renders an ischaemic/thrombotic mechanism more likely. The diagnosis is supported by contrast-enhanced MRI showing optic nerve enhancement in 60-70\%, while brain MRI abnormalities are also common (67\%). Pulse intravenous methylprednisolone in combination with intravenous cyclophosphamide is recommended. ${ }^{25}$ SLE-related optic neuritis is associated with poor visual outcome and only $30 \%$ of patients maintain a visual acuity greater than 20/25. Relapses may occur and merit chronic immunosuppressive therapy. Anticoagulation may be considered in antiphospholipid-positive patients not responding to immunosuppressive therapy.

\section{Peripheral nervous system disorders}

These include polyneuropathy (2-3\%) and less commonly mononeuropathy (single, multiplex), acute inflammatory demyelinating polyradiculoneuropathy, myasthenia gravis, plexopathy, and present with altered sensation, pain, muscle weakness or atrophy. CNS involvement should be excluded by neuroimaging when focal neurological signs, gait disturbance, visual or urinary disorder, increased tendon reflexes and/or muscle tone are present. Nerve conduction studies (NCS) and needle electromyography can identify mononeuropathies, differentiate multiple mononeuropathy versus polyneuropathy and distinguish axonal from demyelinating neuropathies. CSF analysis is useful in inflammatory demyelinating polyradiculoneuropathy. Nerve biopsy is rarely needed to establish the diagnosis. If electrodiagnostic studies are normal, small-fibre neuropathy may be diagnosed by skin biopsy demonstrating loss of intraepidermal nerve fibres. ${ }^{78}$

Glucocorticoids alone or with immunosuppressive therapy have been used with good results (60-75\% response rate). Intravenous immunoglobulin, plasma exchange, and rituximab have been used in severe cases. Peripheral neuropathy has been reported to be a significant predictor of damage in SLE, but a single longitudinal study found that, over a 7-year period, NCS parameters remained unchanged in most $(67 \%)$ patients. $^{79}$

\section{DISCUSSION}

We have developed recommendations for the management of NPSLE patients based upon a systematic review of over 1000 published studies and expert opinion. There is currently no good quality evidence to guide several diagnostic, primary prevention, therapeutic and monitoring decisions in NPSLE, emphasising the need for further research. Nonetheless, consideration of the existing evidence by the expert panel has led to the formulation of NPSLE recommendations with excellent agreement among experts (average 9.1 out of 10 , table 3 ).

We found a considerable variability in reported NPSLE prevalence, which is also due to the rarity of many of the neuropsychiatric syndromes. We categorised NPSLE in order of frequency using estimates of their cumulative incidence based on data from individual studies. After excluding mild neuropsychiatric manifestations, common (cumulative incidence 5-15\%) disorders were CVD and seizures, relatively uncommon (1-5\%) were severe cognitive dysfunction, ACS, psychosis and polyneuropathy, while the remaining neuropsychiatric disorders were rare $(<1 \%)$.

Aetiopathogenic mechanisms involved in NPSLE include vascular injury of intracranial vessels, ${ }^{22} 80$ autoantibodies to neuronal antigens, ribosomes and phospholipid-associated proteins ${ }^{81-83}$ and the intracranial generation of inflammatory mediators. ${ }^{84}$ 85 We identified risk factors associated with NPSLE, as a tool for clinicians to suspect SLE as the underlying cause of a neuropsychiatric event (table 4). General SLE activity or damage, past or concurrent NPSLE and persistently positive antiphospholipid antibodies in moderate-to-high titres were the most significant predictors. These observations provide the rationale for primary and secondary prevention strategies.

In the absence of a diagnostic gold standard for most NPSLE syndromes, numerous serological, CSF and imaging investigations have been used to support the clinical diagnosis. Herein, we have provided estimates of the sensitivity, specificity and diagnostic accuracy of most of these investigations for the diagnosis of various NPSLE syndromes, and have prioritised them according to the clinical setting. Neuroimaging is rapidly evolving and newer brain imaging techniques are increasingly used in NPSLE. Based upon existing evidence and expert opinion, we recommend the use of advanced MRI techniques and/or functional neuroimaging in cases of normal MRI readings or when MRI findings do not correlate with the clinical syndrome. Better biomarkers and neuroimaging tests for SLE-associated neuropsychiatric disease need to be developed that have the capacity to identify the underlying pathological mechanism (ischaemic/ thrombotic vs inflammatory) and guide therapeutic decisions. 
Table 4 Illustrative cases of SLE patients presenting with neuropsychiatric manifestations

Management based on EULAR recommendations

\section{Case 1}

48-Year-old woman with SLE and history of single seizure; serology notable for anti-ds DNA (+), antiphospholipid (-)

Presents with progressive memory decline and difficulties with speech and complex task completion

Non-CNS SLEDAl: 8; Brain MRI: periventricular WM focal T2-weighted hyperintensities

Case 2

38-Year-old woman with history of SLE nephritis (class III); serology: anti-dsDNA

$(+)$, antiphospholipid (-)

Smoker, dyslipidemia (LDL-C $150 \mathrm{mg} / \mathrm{dl}$ )

Presents with right hemiparesis and motor aphasia

Modest lupus activity (SLEDAl: 6)

Case 3

16-Year-old woman presents with fever and generalised tonic-clonic seizures/status epilepticus

CSF examination with mild pleocytosis and increased protein; negative gram stain and PCR for HSV

Arthritis, malar rash, alopecia, lymphadenopathy

Serology: ANA 1:640, anti-dsDNA (+), antiphospholipid (-)

Case 4

31-Year-old woman with SLE. Major depression progressively deteriorating for the last year interfering with work. Mild SLE activity (malar rash, mild arthritis)

Case 5

28-Year-old woman with SLE and several weeks of new-onset headaches of moderate severity. No focal neurological signs or fever. Clinical: arthritis, malar rash

Now presents with tingling sensation in the hands in the absence of arthritis; antiphospholipid (-)

\section{Case 6}

23-Year-old man with SLE, mild arthralgias and a previous history of discoid rash and serositis

He presents with left hemiparesis. No history of hypertension or smoking. Serology: anti-DNA (-), C3, C4: normal, LAC: positive, aCL IgG: 55 GPL, no hyperlipidemia
This patient has possible cognitive dysfunction. Risk factors include previous major NPSLE (seizure) and generalised SLE activity. Neuropsychological testing (1 h ACR battery) diagnosed moderate cognitive dysfunction. Brain MRI was performed due to the young age and the progressive cognitive decline reported by the patient. She received glucocorticoids and azathioprine due to generalised SLE activity and brain MRI lesions. Follow-up neuropsychological examination after 6 months.

This patient has ischaemic stroke. Brain MRI with DWI showed ischaemic lesions (left middle cerebral artery) and the MRA revealed thrombosis of the left internal carotid and the left middle cerebral arteries. Carotid artery ultrasound showed mild atherosclerotic lesions. The patient was started on aspirin and statin, and she was advised to stop smoking. Blood pressure was controlled by ACE-inhibitor. Disease activity was controlled with low-dose glucocorticoids and hydroxychloroquine.

This patient has SLE with seizure disorder. Brain MRI was normal and the EEG revealed epileptiform activity. The patient received anti-epileptic therapy (phenobarbital), and glucocorticoids, hydroxychloroquine, and azathioprine to control disease activity. She was scheduled for a follow-up EEG to guide duration of AED therapy.

This patient has mood disorder (major depression) with poor response to various antidepressants. Brain MRI—performed due to her severe, progressive symptoms—was normal. Disease activity was controlled with glucocorticoids and azathioprine with moderate improvement in her depression.

This patient has headache with some concerning features (new-onset, persistent moderate severity). Brain MRI showed subcortical WM focal hyperintensities. The patient received short-course of low-dose glucocorticoids with clinical improvement.

Needle electromyography and NCS were unremarkable. Spinal cord MRI revealed longitudinal myelitis (C2-C7, T1). Mild CSF abnormalities (7 cells/hpf, protein $66 \mathrm{mg} / \mathrm{dl}$, IgG index (-)). The patient received pulses IV-MP and immunosuppressive therapy.

This patient has stroke in the context of SLE-related APS. Brain MRI revealed infarct in the right parietal lobe. Treated with oral anticoagulants (target INR 3.1-4). Low dose of glucocorticoids was continued with gradual tapering and hydroxychloroquine was added. After 3 years of follow-up, no new cerebrovascular events were reported.

aCL, anticardiolipin antibodies; AED, anti-epileptic drug; ANA, antinuclear antibody; APS, antiphospholipid syndrome; CSF, cerebrospinal fluid; CNS, central nervous system; DWI diffusion-weighted imaging; EULAR, European League Against Rheumatism; GPL, IgG anticardiolipin unit (1 GPL units $=1 \mu \mathrm{g}$ affinity-purified lgG ACA from an original index serum sample): HSV, herpes simplex virus; INR, international normalised ratio; IV-MP, intravenous methylprednisolone; LAC, lupus anticoagulant: LDL-C, low-density lipoprotein cholesterol; MRA, magnetic resonance angiography; NCS, nerve conduction studies; NPSLE, neuropsychiatric systemic lupus erythematosus; SLE, systemic lupus erythematosus; SLEDAI, SLE disease activity index; WM, white matter

Current therapeutic strategies in NPSLE include the use of immunosuppressive therapies when the underlying pathogenesis is considered primarily inflammatory or there is evidence of generalised SLE activity, antiplatelet/antithrombotic therapy when persistently positive moderate-to-high titres of antiphospholipid antibodies or other APS features are present, appropriate symptomatic interventions as indicated, and the treatment of non-SLE factors. The effectiveness of many interventions, however, should be further defined in future RCT.

Clinical practice recommendations require a framework to assess their quality, and to ensure that potential biases have been adequately addressed, are both internally and externally valid, and that are feasible in daily practice. We have ensured that the current guidelines fulfill satisfactorily the AGREE instrument. Following this first round of recommendations, we intend to update them every 3 years with the inclusion of patients and individuals from other relevant professions, and the development of tools that will facilitate the dissemination and implementation of the recommendations.

Author affiliations ${ }^{1}$ Rheumatology, Clinical Immunology and Allergy, University of Crete, Heraklion, Greece

${ }^{2}$ Clinical Trials and Evidence-Based Medicine Unit, Department of Hygiene and Epidemiology, University of loannina School of Medicine, Ioannina, Greece
${ }^{3}$ Department of Medicine III, Division of Rheumatology, University Medical Center Carl Gustav Carus, Technical University of Dresden, Dresden, Germany

${ }^{4}$ Division of Neurology, Leiden University Medical Center, Leiden, The Netherlands;

${ }^{5}$ Cattedra di Reumatologia, Universita di Pisa, Pisa, Italy

${ }^{6}$ Arc Epidemiology Unit, Manchester Academic Health Sciences Centre, The

University of Manchester, Manchester, UK

${ }^{7}$ Department of Autoimmune Diseases, Hospital Clínic, Barcelona, Catalonia, Spain

${ }^{8}$ Clinical Neurosciences, Neuromuscular Diseases, Imperial College, London, UK

${ }^{9}$ Division of Rheumatology, Clinical and Experimental Medicine, University of Padova,

Padova, Italy

${ }^{10}$ Division of Rheumatology, Department of Medicine and Department of Pathology, Capital Health and Dalhousie University, Halifax, Canada

${ }^{11}$ Department of Rheumatology, Leiden University Medical Center, Leiden, The Netherlands

${ }^{12}$ Centre for Rheumatology, University College London Hospitals, London, UK

${ }^{13}$ Department of Rheumatology and Clinical Immunology, University Medical Center

Groningen, Groningen, The Netherlands

${ }^{14}$ Service de médecine interne, centre hospitalier universitaire Pitié-Salpêtrière,

Université Pierre-et-Marie-Curie, Paris, France

${ }^{15}$ Rheumatology, Heinrich-Heine-University, Düsseldorf, Germany

${ }^{16}$ Institute of Clinical Neurosciences, Frenchay Hospital, Bristol, UK

${ }^{17}$ Department of Rheumatology, Medical University of Vienna, Vienna, Austria

${ }^{18}$ Patient representative

${ }^{19}$ Hospital for Special Surgery, New York, USA

${ }^{20}$ Division of Rheumatology, University of Athens, Athens, Greece

${ }^{21}$ Rheumatology and Clinical Immunology, Spedali Civili and University of Brescia,

Brescia, Italy

${ }^{22}$ Department of Radiology, Leiden University Medical Center, Leiden, The Netherlands 
${ }^{23}$ Rheumatology Unit, Department of Medicine, Karolinska University, Stockholm Sweden

${ }^{24}$ National Institute of Arthritis and Musculoskeletal and Skin Diseases, National Institutes of Health, Bethesda, Maryland, USA

${ }^{25}$ Centre for Immune Regulation, School of Immunity and Infection, The University of Birmingham, Birmingham, UK

Acknowledgements The committee wishes to acknowledge the support of the EULAR Executive Committee and Alan Tyndall, the chairman of the Standing Committee for Clinical Affairs. The committee expresses its sincere appreciation and gratitude to the EULAR secretariat and especially to Anja Schönbächler, executive assistant, for their warm hospitality and outstanding organisation.

Funding This study was funded by the European League Against Rheumatism.

Provenance and peer review Not commissioned; externally peer reviewed.

\section{REFERENCES}

1. The American College of Rheumatology nomenclature and case definitions for neuropsychiatric lupus syndromes. Arthritis Rheum 1999;42:599-608.

2. Bertsias G, loannidis JP, Boletis J, et al EULAR recommendations for the management of systemic lupus erythematosus. Report of a task force of the EULAR Standing Committee for International Clinical Studies including therapeutics. Ann Rheum Dis 2008;67:195-205.

3. Ainiala H, Hietaharju A, Loukkola J, et al Validity of the new American College of Rheumatology criteria for neuropsychiatric lupus syndromes: a population-based evaluation. Arthritis Rheum 2001;45:419-23.

4. Hanly JG, Su L, Farewell V, et al Prospective study of neuropsychiatric events in systemic lupus erythematosus. J Rheumatol 2009;36:1449-59.

5. Andrade RM, Alarcón GS, González LA, et al Seizures in patients with systemic lupus erythematosus: data from LUMINA, a multiethnic cohort (LUMINA LIV). Ann Rheum Dis 2008;67:829-34.

6. Mikdashi J, Handwerger B. Predictors of neuropsychiatric damage in systemic lupus erythematosus: data from the Maryland lupus cohort. Rheumatology (Oxford) 2004;43:1555-60.

7. Tomietto P, Annese V, D'Agostini S, et al General and specific factors associated with severity of cognitive impairment in systemic lupus erythematosus. Arthritis Rheum 2007:57:1461-72.

8. Appenzeller S, Cendes F, Costallat LT. Epileptic seizures in systemic lupus erythematosus. Neurology 2004;63:1808-12.

9. Buján S, Ordi-Ros J, Paredes J, et al Contribution of the initial features of systemic lupus erythematosus to the clinical evolution and survival of a cohort of Mediterranean patients. Ann Rheum Dis 2003:62:859-65.

10. Mikdashi J, Krumholz A, Handwerger B. Factors at diagnosis predict subsequent occurrence of seizures in systemic lupus erythematosus. Neurology 2005;64:2102-7

11. Sanna G, Bertolaccini ML, Cuadrado MJ, et al Neuropsychiatric manifestations in systemic lupus erythematosus: prevalence and association with antiphospholipid antibodies. J Rheumatol 2003;30:985-92.

12. Mok MY, Chan EY, Fong DY, et al Antiphospholipid antibody profiles and their clinical associations in Chinese patients with systemic lupus erythematosus. J Rheumatol 2005;32:622-8.

13. Kozora E, West SG, Kotzin BL, et a/ Magnetic resonance imaging abnormalities and cognitive deficits in systemic lupus erythematosus patients without overt central nervous system disease. Arthritis Rheum 1998;41:41-7.

14. Nomura K, Yamano S, Ikeda Y, et al Asymptomatic cerebrovascular lesions detected by magnetic resonance imaging in patients with systemic lupus erythematosus lacking a history of neuropsychiatric events. Intern Med 1999;38:785-95.

15. Sibbitt WL, Jr, Sibbitt RR, Griffey RH, et al Magnetic resonance and computed tomographic imaging in the evaluation of acute neuropsychiatric disease in systemic lupus erythematosus. Ann Rheum Dis 1989;48:1014-22.

16. Appenzeller S, Li LM, Costallat LT, et al Evidence of reversible axonal dysfunction in systemic lupus erythematosus: a proton MRS study. Brain 2005;128:2933-40.

17. Axford JS, Howe FA, Heron C, et al Sensitivity of quantitative (1) H magnetic resonance spectroscopy of the brain in detecting early neuronal damage in systemic lupus erythematosus. Ann Rheum Dis 2001;60:106-11.

18. Bosma GP, Steens SC, Petropoulos H, et al Multisequence magnetic resonance imaging study of neuropsychiatric systemic lupus erythematosus. Arthritis Rheum 2004;50:3195-202.

19. Steens SC, Admiraal-Behloul F, Bosma GP, et al Selective gray matter damage in neuropsychiatric lupus. Arthritis Rheum 2004;50:2877-81.

20. Hughes $\mathbf{M}$, Sundgren PC, Fan X, et al Diffusion tensor imaging in patients with acute onset of neuropsychiatric systemic lupus erythematosus: a prospective study of apparent diffusion coefficient, fractional anisotropy values, and eigenvalues in different regions of the brain. Acta Radiol 2007;48:213-22.

21. Castellino G, Padovan M, Bortoluzzi A, et al Single photon emission computed tomography and magnetic resonance imaging evaluation in SLE patients with and without neuropsychiatric involvement. Rheumatology (Oxford) 2008;47:319-23.
22. Zhang $\mathbf{X}$, Zhu Z, Zhang F, et al Diagnostic value of single-photon-emission computed tomography in severe central nervous system involvement of systemic lupus erythematosus: a case-control study. Arthritis Rheum 2005;53:845-9.

23. Weiner SM, Otte A, Schumacher M, et al Diagnosis and monitoring of central nervous system involvement in systemic lupus erythematosus: value of $\mathrm{F}-18$ fluorodeoxyglucose PET. Ann Rheum Dis 2000;59:377-85

24. Stojanovich L, Stojanovich R, Kostich V, et al Neuropsychiatric lupus favourable response to low dose i.v. cyclophosphamide and prednisolone (pilot study). Lupus 2003;12:3-7.

25. Barile-Fabris L, Ariza-Andraca R, Olguín-Ortega L, et al Controlled clinical trial of IV cyclophosphamide versus IV methylprednisolone in severe neurological manifestations in systemic lupus erythematosus. Ann Rheum Dis 2005;64:620-5.

26. Neuwelt CM. The role of plasmapheresis in the treatment of severe central nervous system neuropsychiatric systemic lupus erythematosus. Ther Apher Dial 2003; 7:173-82.

27. Bartolucci P, Bréchignac $\mathrm{S}$, Cohen $\mathrm{P}$, et al Adjunctive plasma exchanges to treat neuropsychiatric lupus: a retrospective study on 10 patients. Lupus 2007;16:817-22.

28. Levy Y, Sherer Y, Ahmed A, et al A study of 20 SLE patients with intravenous immunoglobulin - clinical and serologic response. Lupus 1999;8:705-12.

29. Milstone AM, Meyers K, Elia J. Treatment of acute neuropsychiatric lupus with intravenous immunoglobulin (IVIG): a case report and review of the literature. Clin Rheumatol 2005;24:394-7.

30. Vina ER, Fang AJ, Wallace DJ, et al Chronic inflammatory demyelinating polyneuropathy in patients with systemic lupus erythematosus: prognosis and outcome. Semin Arthritis Rheum 2005:35:175-84.

31. Tokunaga M, Saito K, Kawabata D, et al Efficacy of rituximab (anti-CD20) for refractory systemic lupus erythematosus involving the central nervous system. Ann Rheum Dis 2007;66:470-5

32. Khamashta MA, Cuadrado MJ, Mujic F, et al The management of thrombosis in the antiphospholipid-antibody syndrome. N Engl J Med 1995;332:993-7.

33. Muñoz-Rodriguez FJ, Font J, Cervera R, et al Clinical study and follow-up of 100 patients with the antiphospholipid syndrome. Semin Arthritis Rheum 1999;29:182-90.

34. Tektonidou MG, loannidis JP, Boki KA, et al Prognostic factors and clustering of serious clinical outcomes in antiphospholipid syndrome. O J Med 2000;93:523-30.

35. Ruiz-Irastorza G, Hunt BJ, Khamashta MA. A systematic review of secondary thromboprophylaxis in patients with antiphospholipid antibodies. Arthritis Rheum 2007; 57:1487-95.

36. Cervera R, Asherson RA, Font J, et a/ Chorea in the antiphospholipid syndrome. Clinical, radiologic, and immunologic characteristics of 50 patients from our clinics and the recent literature. Medicine (Baltimore) 1997;76:203-12.

37. D'Cruz DP, Mellor-Pita S, Joven B, et al Transverse myelitis as the first manifestation of systemic lupus erythematosus or lupus-like disease: good functional outcome and relevance of antiphospholipid antibodies. J Rheumatol 2004;31:280-5.

38. Heinlein AC, Gertner E. Marked inflammation in catastrophic longitudinal myelitis associated with systemic lupus erythematosus. Lupus 2007;16:823-6.

39. Tarr T, Lakos G, Bhattoa HP, et al Analysis of risk factors for the development of thrombotic complications in antiphospholipid antibody positive lupus patients. Lupus 2007:16:39-45

40. Hereng $\mathbf{T}$, Lambert $M$, Hachulla $\mathrm{E}$, et al Influence of aspirin on the clinical outcomes of 103 anti-phospholipid antibodies-positive patients. Lupus 2008;17:11-15

41. Tektonidou MG, Laskari K, Panagiotakos DB, et al Risk factors for thrombosis and primary thrombosis prevention in patients with systemic lupus erythematosus with or without antiphospholipid antibodies. Arthritis Rheum 2009;61:29-36.

42. Erkan D, Harrison MJ, Levy R, et al Aspirin for primary thrombosis prevention in the antiphospholipid syndrome: a randomized, double-blind, placebo-controlled trial in asymptomatic antiphospholipid antibody-positive individuals. Arthritis Rheum 2007:56:2382-91

43. Mitsikostas DD, Sfikakis PP, Goadsby PJ. A meta-analysis for headache in systemic lupus erythematosus: the evidence and the myth. Brain 2004;127:1200-9.

44. Crowther MA, Ginsberg JS, Julian J, et al A comparison of two intensities of warfarin for the prevention of recurrent thrombosis in patients with the antiphospholipid antibody syndrome. N Engl J Med 2003;349:1133-8.

45. Finazzi G, Marchioli R, Brancaccio V, et al A randomized clinical trial of high-intensity warfarin vs. conventional antithrombotic therapy for the prevention of recurrent thrombosis in patients with the antiphospholipid syndrome (WAPS). J Thromb Haemost 2005;3:848-53.

46. Ruiz-Irastorza G, Khamashta MA, Hunt BJ, et al Bleeding and recurrent thrombosis in definite antiphospholipid syndrome: analysis of a series of 66 patients treated with oral anticoagulation to a target international normalized ratio of 3.5. Arch Intern Med 2002;162:1164-9.

47. Finazzi G, Brancaccio V, Moia M, et al Natural history and risk factors for thrombosis in 360 patients with antiphospholipid antibodies: a four-year prospective study from the Italian Registry. Am J Med 1996;100:530-6.

48. Cervera R, Boffa MC, Khamashta MA, et al The Euro-Phospholipid Project: epidemiology of the antiphospholipid syndrome in Europe. Lupus 2009;18:889-93.

49. Cohen D, Berger SP, Steup-Beekman GM, et al Diagnosis and management of the antiphospholipid syndrome. BMJ 2010;340:c2541. 
50. Panopalis $\mathbf{P}$, Julian L, Yazdany J, et al Impact of memory impairment on employment status in persons with systemic lupus erythematosus. Arthritis Rheum 2007:57:1453-60.

51. Ainiala H, Dastidar P, Loukkola J, et al Cerebral MRI abnormalities and their association with neuropsychiatric manifestations in SLE: a population-based study. Scand J Rheumatol 2005;34:376-82.

52. Lapteva L, Nowak M, Yarboro CH, et al Anti-N-methyl-d-aspartate receptor antibodies, cognitive dysfunction, and depression in systemic lupus erythematosus. Arthritis Rheum 2006;54:2505-14.

53. Waterloo K, Omdal R, Sjöholm H, et al Neuropsychological dysfunction in systemic lupus erythematosus is not associated with changes in cerebral blood flow. J Neurol 2001;248:595-602.

54. McLaurin EY, Holliday SL, Williams P, et al Predictors of cognitive dysfunction in patients with systemic lupus erythematosus. Neurology 2005;64:297-303.

55. Harrison MJ, Morris KA, Horton R, et al Results of intervention for lupus patients with self-perceived cognitive difficulties. Neurology 2005;65:1325-7.

56. Haupt M, Millen S, Jänner M, et al Improvement of coping abilities in patients with systemic lupus erythematosus: a prospective study. Ann Rheum Dis 2005;64:1618-23.

57. Hanly JG, Cassell K, Fisk JD. Cognitive function in systemic lupus erythematosus results of a 5-year prospective study. Arthritis Rheum 1997:40:1542-3.

58. Denburg SD, Carbotte RM, Denburg JA. Corticosteroids and neuropsychological functioning in patients with systemic lupus erythematosus. Arthritis Rheum 1994;37:1311-20.

59. Hughes GR, Cuadrado MJ, Khamashta MA, et al Headache and memory loss: rapid response to heparin in the antiphospholipid syndrome. Lupus 2001;10:778.

60. González-Duarte A, Cantú-Brito CG, Ruano-Calderón L, et al Clinical description of seizures in patients with systemic lupus erythematosus. Eur Neurol 2008;59:320-3.

61. Joseph FG, Lammie GA, Scolding NJ. CNS lupus: a study of 41 patients. Neurology 2007;69:644-54

62. Orzechowski NM, Wolanskyj AP, Ahlskog JE, et al Antiphospholipid antibodyassociated chorea. J Rheumatol 2008;35:2165-70.

63. Dennis MS, Byrne EJ, Hopkinson N, et al Neuropsychiatric systemic lupus erythematosus in elderly people: a case series. J Neurol Neurosurg Psychiatr 1992;55:1157-61.

64. Rahman P, Humphrey-Murto S, Gladman DD, et al Cytotoxic therapy in systemic lupus erythematosus. Experience from a single center. Medicine (Baltimore) 1997; 76:432-7.

65. Chau SY, Mok CC. Factors predictive of corticosteroid psychosis in patients with systemic lupus erythematosus. Neurology 2003;61:104-7.

66. Briani C, Lucchetta M, Ghirardello A, et al Neurolupus is associated with anti-ribosomal P protein antibodies: an inception cohort study. J Autoimmun 2009;32:79-84.

67. Hanly JG, Urowitz MB, Siannis F, et al Autoantibodies and neuropsychiatric events at the time of systemic lupus erythematosus diagnosis: results from an internationa inception cohort study. Arthritis Rheum 2008;58:843-53

68. Karassa FB, Afeltra A, Ambrozic A, et al Accuracy of anti-ribosomal P protein antibody testing for the diagnosis of neuropsychiatric systemic lupus erythematosus: an international meta-analysis. Arthritis Rheum 2006;54:312-24.
69. Kodama K, Okada S, Hino T, et al Single photon emission computed tomography in systemic lupus erythematosus with psychiatric symptoms. J Neurol Neurosurg Psychiatr 1995:58:307-11.

70. Greco CM, Rudy TE, Manzi S. Effects of a stress-reduction program on psychological function, pain, and physical function of systemic lupus erythematosus patients: a randomized controlled trial. Arthritis Rheum 2004;51:625-34.

71. Martin-Suarez I, D'Cruz D, Mansoor M, et al Immunosuppressive treatment in severe connective tissue diseases: effects of low dose intravenous cyclophosphamide. Ann Rheum Dis 1997:56:481-7.

72. Mok CC, Lau CS, Wong RW. Treatment of lupus psychosis with oral cyclophosphamide followed by azathioprine maintenance: an open-label study. Am J Med 2003;115:59-62

73. Pego-Reigosa JM, Isenberg DA. Psychosis due to systemic lupus erythematosus: characteristics and long-term outcome of this rare manifestation of the disease. Rheumatology (Oxford) 2008;47:1498-502.

74. Birnbaum J, Petri M, Thompson R, et al Distinct subtypes of myelitis in systemic lupus erythematosus. Arthritis Rheum 2009;60:3378-87.

75. Pittock SJ, Lennon VA, de Seze J, et al Neuromyelitis optica and non organ-specific autoimmunity. Arch Neurol 2008;65:78-83

76. Kovacs B, Lafferty TL, Brent LH, et al Transverse myelopathy in systemic lupus erythematosus: an analysis of 14 cases and review of the literature. Ann Rheum Dis 2000;59:120-4.

77. Lu X, Gu Y, Wang Y, et al Prognostic factors of lupus myelopathy. Lupus 2008;17:323-8.

78. Tseng MT, Hsieh SC, Shun CT, et al Skin denervation and cutaneous vasculitis in systemic lupus erythematosus. Brain 2006;129:977-85.

79. Omdal R, Løseth S, Torbergsen T, et al Peripheral neuropathy in systemic lupus erythematosus - a longitudinal study. Acta Neurol Scand 2001;103:386-91.

80. Emmer BJ, Steup-Beekman GM, Steens SC, et al Correlation of magnetization transfer ratio histogram parameters with neuropsychiatric systemic lupus erythematosus criteria and proton magnetic resonance spectroscopy: association of magnetization transfer ratio peak height with neuronal and cognitive dysfunction. Arthritis Rheum 2008:58:1451-7.

81. Kowal C, Degiorgio LA, Lee JY, et al Human lupus autoantibodies against NMDA receptors mediate cognitive impairment. Proc Natl Acad Sci USA 2006;103:19854-9.

82. Matus S, Burgos PV, Bravo-Zehnder M, et al Antiribosomal-P autoantibodies from psychiatric lupus target a novel neuronal surface protein causing calcium influx and apoptosis. J Exp Med 2007;204:3221-34.

83. Zhang $\mathbf{X}$, Shu $\mathrm{H}$, Zhang $\mathrm{F}$, et al Cell-ELISA detection of antineuronal antibodies in central nervous system involvement in systemic lupus erythematosus. Ann Rheum Dis 2007:66:530-2.

84. Fragoso-Loyo H, Richaud-Patin Y, Orozco-Narváez A, et al Interleukin-6 and chemokines in the neuropsychiatric manifestations of systemic lupus erythematosus. Arthritis Rheum 2007;56:1242-50.

85. Trysberg $\mathbf{E}$, Blennow K, Zachrisson 0 , et al Intrathecal levels of matrix metalloproteinases in systemic lupus erythematosus with central nervous system engagement. Arthritis Res Ther 2004;6:R551-6. 